\title{
Variação de conduta cirúrgica em doença degenerativa da coluna lombar
}

\author{
Surgical decision making variation in degenerative lumbar spine \\ Variación de conducta quirúrgica en la enfermedad degenerativa \\ de la columna lumbar
}

\author{
Luís Felipe Pamplona Novaes ${ }^{1}$ \\ Marcelo Ítalo Risso Neto ${ }^{2}$ \\ Ivan Guidolin Veiga ${ }^{2}$ \\ Wagner Pasqualini ${ }^{2}$ \\ Paulo Tadeu Maia Cavali² \\ Elcio Landim ${ }^{3}$
}

\section{RESUMO}

Objetivo: examinar a variação da decisão de tratamento entre cirurgiões de coluna para cinco situações clínicas que envolvam condições degenerativas da coluna lombar e determinar se fatores como tipo de treinamento do cirurgião, experiência e idade são como preditores da tomada de decisões para cada caso. Métodos: dez ortopedistas e dez neurocirurgiões, de diferentes idades, responderam questões sobre a necessidade de cirurgia, opções de via de acesso, realização de descompressão, realização de artrodese, com ou sem instrumentação, para cinco casos de doença degenerativa da coluna lombar por meio de informações da história, exame clínico e exames de imagem adequados (todos tiveram acesso às mesmas informações e foram orientados a responder com base em sua prática clínica). Os casos foram: disco-

\section{ABSTRACT}

Objective: to determine the surgical decision making variation in five degenerative lumbar spine clinical situations. Determine whether factor related to spinal surgeon training program, experience and age are determinants in surgical decision making for each case. Methods: ten orthopedists and ten neurosurgeons from different ages were questioned about surgery indication, approach options, need for decompression, need for fusion with instrumentation or not for five degenerative lumbar spinal clinical scenarios. Information about history, examination and image studies (all examiners had the same information and oriented to answer thinkingintheirownexperience). Cases presented:instabilityanddiscdiseasein a Youngpatient; extrudedlumbarhérnia

\section{RESUMEN}

Objetivos: examinar la variación de la decisión de tratamiento entre cirujanos de la columna para cinco escenarios clínicos involucrando condiciones degenerativas de la columna lumbar. Determinar si el tipo del entrenamiento del cirujano, experiencia y edad sirve como factores predictivos en la toma de decisiones de cada caso. Métodos: diez ortopedistas y diez neurocirujanos de diferentes edades respondieron un cuestionario sobre la necesidad de cirugía, opciones de vías de acceso, realización de descompresión, realización de artrodesis con o sin instrumentación para 5 casos de enfermedad degenerativa de la columna lumbar por medio de informaciones de la historia, examen clínico y exámenes de imagen adecuados (todos tuvieron acceso a las mismas informaciones yorientados a responder basados

\footnotetext{
Trabalho realizado na Disciplina de Cirurgia de Coluna do Departamento de Ortopedia e Traumatologia da Faculdade de Ciências Médicas da Universidade Estadual de Campinas - UNICAMP - Campinas (SP), Brasil.

'Estagiário do Curso de Especialização em Cirurgia da Coluna da Faculdade de Ciências Médicas da Universidade Estadual de Campinas - UNICAMP - Campinas (SP), Brasil.

${ }^{2}$ Assistente Grupo de Coluna da Faculdade de Ciências Médicas da Universidade Estadual de Campinas - UNICAMP - Campinas (SP), Brasil.

${ }^{3}$ Professor, Chefe Grupo de Coluna da Faculdade de Ciências Médicas da Universidade Estadual de Campinas - UNICAMP - Campinas (SP), Brasil. 
patia com instabilidade vertebral em um paciente jovem; hérnia de disco extrusa em paciente jovem; estenose de canal vertebral múltiplos níveis sem deformidade; estenose canal múltiplos níveis com cifoescoliose degenerativa; hérnia de disco com artrose facetária. Na análise estatística foi utilizado o teste " $\mathrm{t}$ " de student para comparar o fator especialidade e médias de idade com as variáveis de tratamento (significativo quando $\mathrm{p}<0,05)$. Resultados: a média de idade dos pacientes era de 42,15 anos (variando de 29 a 56 anos). Dos pacientes entrevistados, 12 (60\%) são de origem do Estado de São Paulo e oito (40\%) de outros Estados. Não foram observadas variações significativas para diferentes abordagens. De um modo geral, os ortopedistas recomendam a realização de artrodese e instrumentação com mais frequência que os neurocirurgiões, com significância maior no caso de escoliose degenerativa com estenose de canal $(p=0,04)$ e também no caso de hérnia discal $(p=0,01)$. Com relação a idade, cirurgiões mais experientes optaram mais pela instrumentação que os mais jovens no caso de estenose e instabilidade sem deformidade $(\mathrm{p}=0,001)$. Conclusão: há concordância quanto às preferências dos cirurgiões de coluna, sendo que as diferenças restringem-se a preferência pela instrumentação entre os ortopedistas quando confrontados com casos de hérnia de disco lombar, bem como o tratamento não apenas da clínica neurológica, mas também da deformidade no caso de escoliose degenerativa. A idade dos cirurgiões e o tempo de atividade foram pouco determinantes nas indicações cirúrgicas. Há crescente uniformização no treinamento dos cirurgiões de coluna, restandodiferençasqueremontam talvez a sua formação básica, entre ortopedistas e neurocirurgiões.

DESCRITORES: Fusão vertebral; Coluna vertebral/ cirurgia; Escoliose; Hérnia discal; Doenças da coluna vertebral in ayoung patient; multiplelevel spinal stenosis without deformity; multiple spinal stenosis with degenerative kyphoscoliosis; lumbar hernia with facet degeneration. Effects on training background and surgeon mean age on surgical decision making were analyzed using Student " $t$ " test $(p<0.05)$. Results: mean age was 42.15 years (varying 29 to 56 years). Twelve (60\%) participants were from Sao Paulo while resting eight $(40 \%)$ were from other states. Significative variation was notobserved. In a general manner, orthopedists recommend fusion and instrumentation more often then neurosurgeons, reaching significance for degenerative scoliosis with spinal stenosis $(p=0.04)$ and for lumbar hernia $(p=0.01)$. Experienced surgeons were more likely to recommend instrumentations than younger for spinal stenosis and instability without deformity ( $p=0.001$ ). Conclusion: there is a great agreement about surgeon's preferences, resting differences in orthopedists preference for instrumentation for lumbar disc herniation cases and treatment of the deformity for degenerative scoliosis with spinal stenosis case. Surgeon's age and experience were not determinant in surgical decision making. Actually there is a growing uniformization in spinal surgeon training programs with a few differences inherent to basic training for orthopedists and neurosurgeons.

KEYWORDS: Spinal fusion; Spine/ surgery; Scoliosis; Intervertebral disc displacement; Spinal disease en su práctica clínica). Los casos fueron: discopatía con inestabilidad vertebral en un paciente joven; hernia de disco extrusa en paciente joven; estenosis canal vertebral múltiples niveles sin deformidad; estenosis canal múltiples niveles con cifoescoliosis degenerativa; hernia discal con artrosis facetaria. El análisis estadístico utilizado fue el test " $t$ " de Student para comparar el factor especialidad y promedios de edad con las variables de tratamiento (significativo con $p<0.05$ ). Resultados: el promedio de edad fue de 42.15 años (variando de 29 a 56 años). Doce (60\%) entrevistados son originarios del Estado de Sao Paulo y los ocho (40\%) restantes fueron de otros estados. Variaciones significativas para diferentes abordajes no fueron observadas. De un modo general, los ortopedistas recomiendan la artrodesis e instrumentación con más frecuencia que los neurocirujanos, con una frecuencia significativa en caso de escoliosis degenerativa con estenosis de canal ( $p=0.04)$ y también en el caso de hernia discal $(p=0.01)$. Con relación a la edad, cirujanos con mayor experiencia optaron más por la instrumentación que los más jóvenes en el caso de estenosis e inestabilidad sin deformidad ( $p=0.001)$. Conclusión: hay una gran concordancia en cuanto a las preferencias de los cirujanos de columna, siendo que las diferencias fueron restringidas a la preferencia por la instrumentación entre los ortopedistas cuando enfrentados con casos de hernia de disco lumbar; así como un tratamiento no solo de la clínica neurológica sino también de la deformidad en el caso de la escoliosis degenerativa. La edad de los cirujanos $y$ el tiempo de actividad fueron poço determinantes en las indicaciones quirúrgicas. Hay una uniformidad creciente en el entrenamiento de los cirujanos de columna, restando diferencias que se remontan tal vez a la formación básica, entre ortopedistas y neurocirujanos.

DESCRIPTORES: Fusión vertebral; Columna vertebral/cirugía; Escoliosis; Desplazamiento del disco intervertebral; Enfermedades de la columna vertebral 


\section{INTRODUÇÃO}

Há uma variação significativa quanto ao tratamento de diversas situações clínicas entre diferentes médicos, quanto a procedimentos como bypass de coronária, histerectomia, prostatectomia, tratamento do infarto agudo do miocárdio com índices que variam em até dez vezes entre os profissionais 1-4. Também foram observadas variações entre procedimentos cirúrgicos ortopédicos como artroscopia de joelho e ombro, artroplastia de quadril e joelho e quanto a fratura do fêmur proxima ${ }^{15-8}$. Ao mesmo tempo, as indicações de cirurgia eletiva para as doenças degenerativas da coluna lombar variam bastante, com diferenças nos índices de laminectomia e artrodese lombar entre sete e dez vezes, respectivamente ${ }^{9}$.

As cirurgias da coluna lombar têm-se mostrado, de uma maneira geral, uniformes entre a maioria dos cirurgiões. Entretanto, outros fatores como idade, estilo de vida, expectativa e estado geral de saúde dos pacientes também podem contribuir para as variações na decisão da conduta cirúrgica $^{7,10-11}$. Fatores inerentes ao cirurgião, como a idade, tempo de experiência com a especialidade e o tipo de treinamento em cirurgia de coluna (ortopédico ou neurocirúrgico) também podem interferir com a abordagem de determinadas situações clínicas.

Há dúvida clínica em vários casos de cirurgia da coluna lombar porque muitas doenças têm múltiplas opções de tratamento com sucesso já descrito e a técnica não é, muitas vezes, reprodutível nas mãos de diferentes cirurgiões ${ }^{12-24}$. Estas incertezas levam a um aumento na confiança no treinamento e experiência clínica pessoal. A análise das diferenças quanto às decisões cirúrgicas em determinadas situações clínicas poderá possibilitar o esclarecimento dos fatores que contribuem para a variação existente.

O objetivo deste estudo é examinar a extensão da variação da decisão de tratamento entre cirurgiões de coluna para cinco situações clínicas que envolvam condições degenerativas da coluna lombar, e determinar, se possível, fatores relacionados ao cirurgião, como tipo de treinamento, experiência e idade, como preditores da tomada de decisões para cada caso apresentado.

\section{MÉTODOS}

Um total de 20 cirurgiões de coluna foram entrevistados, sendo dez ortopedistas e dez neurocirurgiões, todos com treinamento específico e prática diária em cirurgia de coluna. Os participantes foram escolhidos conforme o tipo de treinamento (ortopedistas $\mathrm{x}$ neurocirurgiões) e de diferentes idades. Todos os participantes deveriam analisar e opinar quanto ao tratamento das cinco situações clínicas de doença degenerativa da coluna lombar. Cada apresentação consistia de um caso clínico com história e exame físico e imagens dos estudos radiográficos necessários (raio-X, ressonância magnética, tomografia, conforme a necessidade do caso) dos níveis adequados da coluna lombar. Os observadores tiveram acesso às mesmas informações sobre os casos. Os dados clínicos e de imagem omitidos deveriam ser considerados normais.
Os casos foram selecionados em cinco categorias diferentes de doença degenerativa da coluna lombar:

(1) Discopatia com instabilidade vertebral em um paciente de 32 anos;

(2) Hérnia de disco extrusa em paciente de 37 anos (figuras 1 e 2);

(3) Estenose de canal vertebral em múltiplos níveis, sem deformidade em paciente de 76 anos (figuras 3 e 4);

(4) Estenose de canal em múltiplos níveis com cifoescoliose degenerativa em paciente de 75 anos, ativo;

(5) Hérnia de disco com artrose facetária em paciente de 50 anos.
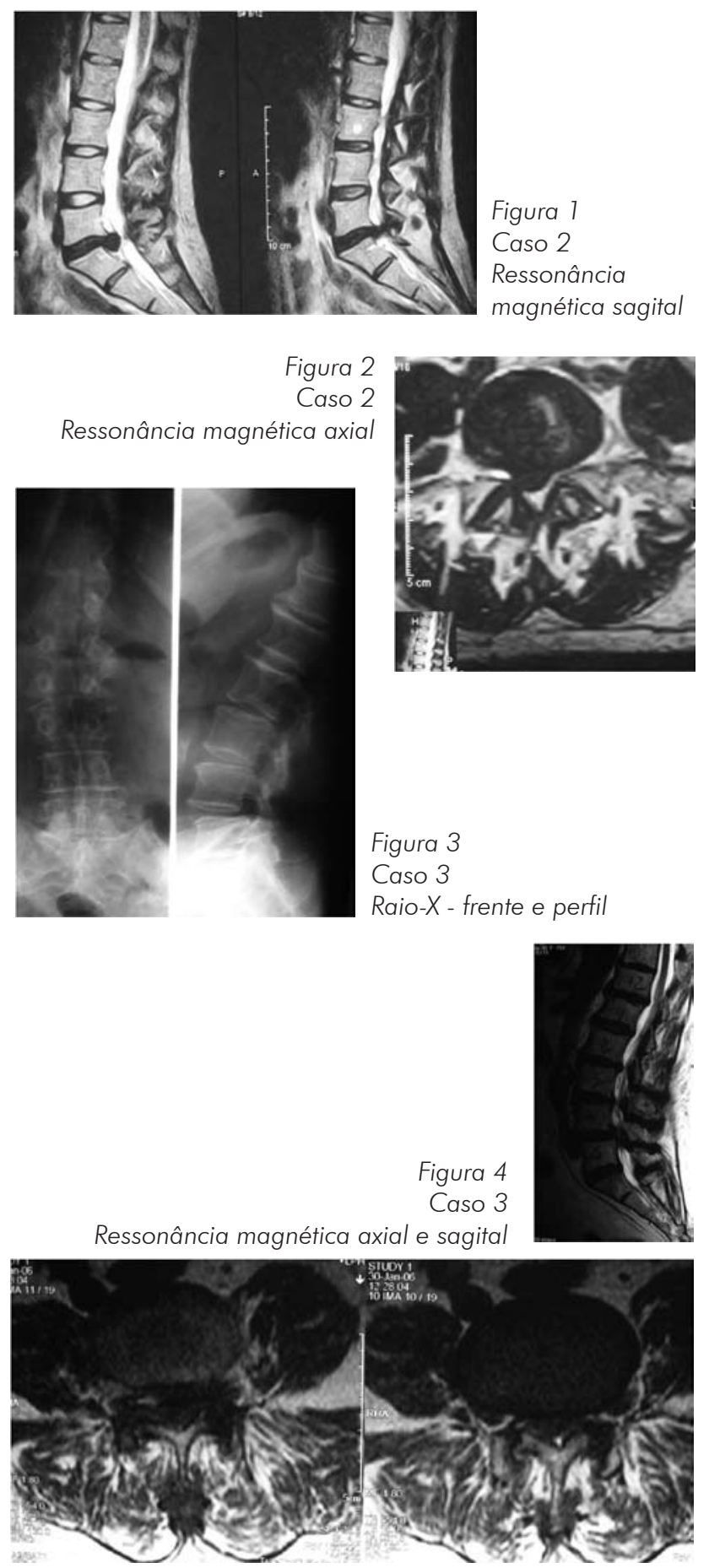
Cada caso foi acompanhado de um questionário padrão com opções relacionadas ao tratamento (cirúrgico). As decisões incluíam: indicação ou não de cirurgia, momento da cirurgia, via de acesso, níveis de descompressão, opção por realização de artrodese ou não, níveis de artrodese e uso de instrumentação. Os participantes foram orientados a não preencher os demais itens do questionário caso decidissem não operar determinado caso. Aqueles que não responderam ao questionário foram excluídos da análise. Também foram solicitados a responder baseados na sua prática clínica e não no que considerariam ser a resposta mais correta. Para fins de análise estatística, foi utilizado o teste " $\mathrm{t}$ " de student para comparar o fator especialidade e médias de idade com as variáveis de tratamento pré-estabelecidas.

\section{RESULTADOS}

Entre os 20 participantes do estudo, a média de idade foi de 42,15 anos (variando de 29 a 56 anos). Entre os ortopedistas a média foi de 38,8 anos (29 a 55) e entre os neurocirurgiões de 45,5 anos ( 33 a 56). Com tempo médio de experiência em cirurgia de coluna de 17,75 anos (variando de 1 a 29 anos). Dos entrevistados, 12 (60\%) eram do Estado de São Paulo e os oito $(40 \%)$ demais de outros estados.

Padrões de resposta para os casos específicos - Para cada um dos cinco casos apresentados, a resposta mais frequente foi indicar a cirurgia no momento inicial. Nem a idade, nem o tipo de especialidade foram determinantes para o momento de indicação cirúrgica para nenhum dos casos.

\section{Caso 1}

No caso da instabilidade intervertebral com discopatia $100 \%$ dos participantes optaram pela cirurgia imediata, assim como a totalidade preferiu a via de acesso posterior. Houve preferência pela instrumentação por nove entre dez ortopedistas $(90 \%)$, sendo uso de sistema dinâmico entre seis entrevistados. Entre os neurocirurgiões, sete $(70 \%)$ optaram por instrumentação, todos por sistemas de parafusos pediculares; o que não apresenta diferença significante $(\mathrm{p}=0,10)$. A realização de artrodese foi indicada por sete $(70 \%)$ dos neurocirurgiões e por apenas três ortopedistas $(\mathrm{p}=0,09)$. Metade de cada grupo optou por descompressão neste caso $(50 \%)$. Pode-se observar diferença significante quando comparada a idade e o tempo de experiência dos cirurgiões e indicação de realização de artrodese, sendo que os cirurgiões mais jovens demonstram preferência pela instrumentação sem a realização de artrodese com a utilização de um sistema dinâmico $(\mathrm{p}=0,01)$.

\section{Caso 2}

No caso de uma hérnia de disco extrusa em paciente jovem, $100 \%$ dos entrevistados indicaram cirurgia imediata, considerando tratamento clínico prévio como fora apresentado o caso. A totalidade de neurocirurgiões e ortopedistas indicaram a descompressão neste caso. No entanto, quatro entre nove (44\%) dos ortopedistas indicaram instrumentação via posterior e realização de artrodese, enquanto que nenhum dos neurocirurgiões fez uso de instrumental ou de realização de artrodese, o que se mostrou estatisticamente significante $(\mathrm{p}=0,01)$.

\section{Caso 3}

No caso de estenose do canal vertebral em múltiplos níveis, com espondilolistese degenerativa, mas sem deformidade, observou-se indicação cirúrgica por todos os participantes. Entre os entrevistados, nove (90\%) ortopedistas e oito $(80 \%)$ neurocirurgiões indicaram a realização de artrodese com instrumentação. Neste caso, podese observar que cirurgiões que indicaram instrumentação eram mais experientes (com média de tempo de cirurgia de coluna de 14 anos) quando comparados aos que não indicaram instrumentação (média de 5,5 anos) $(\mathrm{P}=0,001)$.

\section{Caso 4}

No caso de cifoescoliose degenerativa com estenose de canal em múltiplos níveis, nove $(90 \%)$ ortopedistas e apenas três $(30 \%)$ neurocirurgiões indicaram cirurgia, enquanto os demais não operariam neste caso e foram excluídos da análise. Todos os participantes que indicaram cirurgia também optaram por realizar descompressão. Oito $(89 \%)$ dos ortopedistas e três $(100 \%)$ dos neurocirurgiões, entre os que indicariam cirurgia, indicaram instrumentação e realização de artrodese, sendo que entre os ortopedistas houve concordância em instrumentar toda deformidade enquanto que os neurocirurgiões apenas um segmento da curva. Foi observada uma diferença significante quanto a indicação de cirurgia, havendo preferência entre os ortopedistas quando comparada aos neurocirurgiões $(p=0,04)$. Não houve diferença quando comparada à idade ou tempo de experiência dos cirurgiões.

\section{Caso 5}

Neste caso de hérnia discal com artrose em paciente com mais idade, nove $(90 \%)$ dos neurocirurgiões e sete $(70 \%)$ dos ortopedistas indicaram a cirurgia imediata. Três entre os ortopedistas operariam o caso apenas após mais seis meses de tratamento conservador e observação clínica. Todos os participantes concordam quanto a indicação de descompressão neste caso. Sete entre ortopedistas que responderam ao questionário $(87,5 \%)$ optam por instrumentação e realização de artrodese, enquanto que apenas dois $(22 \%)$ dos neurocirurgiões optaram por esta indicação, o que foi estatisticamente significativo $(\mathrm{p}=0,02)$.

\section{DISCUSSÃO}

Há diversidades entre os tratamentos propostos para diversas situações clínicas embora as explicações para essas variações não tenham sido bem avaliadas. Em nosso estudo, encontramos variações significativas na abordagem terapêutica entre diferentes cirurgiões para determinadas condições clínicas degenerativas da coluna lombar. Por exemplo, no caso no. 3 houve grande concordância quanto à indicação cirúrgica, descompressão, e necessidade de artrodese instrumentada no caso de estenose canal múltiplos níveis com espondilolistese degenerativa associada. Enquanto isso, observamos grande diferença quanto à indicação cirúrgica nos casos de estenose de canal, associada à deformidade, com tendência maior de cirurgia entre ortopedistas. 
Houve grande número de cirurgiões que optaram por não instrumentar ou até mesmo não operar o paciente portador de estenose de canal associada a deformidade (Caso 4). Contra isso, a literatura sugere a realização de artrodese instrumentada para os pacientes com instabilidade no plano coronal ${ }^{12,20}$, embora não tenha respaldo em estudos prospectivos e randomizados. Neste caso, foi observado que o tipo de treinamento foi determinante na escolha da conduta, com os ortopedistas indicando com maior significância o procedimento cirúrgico e a realização de artrodese instrumentada. Este achado pode mostrar uma diferença significativa entre ortopedistas e neurocirurgiões frente a este caso.

No caso 1 de discopatia com instabilidade intervertebral, foi interessante notar o grande número de ortopedistas jovens adeptos de um sistema dinâmico de fixação, enquanto que a maioria $(70 \%)$ dos neurocirurgiões optaram por realização de artrodese. Com relação a este caso, a literatura mostra resultados melhores com a realização de artrodese, seja póstero-lateral, intersomática ou circunferencial, em relação ao tratamento conservador já que o objetivo é eliminar a instabilidade no nível com discopatia ${ }^{25}$. Há relatos que mostram eficácia do sistema dinâmico, com uso de parafusos pediculares conectados a um componente elástico. Assim consegue-se um controle de movimento com a vantagem de não sobrecarregar os níveis adjacentes ${ }^{26-27}$. Estes dados podem apontar mais diferenças no treinamento entre os cirurgiões de coluna de nosso amostra.

Nos casos 2 e 5, na presença de uma hérnia discal, pudemos observar uma nítida preferência pela instrumentação entre os ortopedistas enquanto apenas uma minoria dos neurocirurgiões optaria pela realização de artrodese com instrumental. A discectomia aberta é a cirurgia mais comum, com laminotomia ou uma laminectomia parcial, afastamento das estruturas neurais e excisão conteúdo herniado. Há ainda os que adicionam uso de microscopia, embora não haja benefícios quanto ao resultado clínico. A adição da realização de artrodese aumenta a establidade no segmento e previne a recidiva da hérnia, embora aumente a morbidade do procedimento e pode resultar em discopatia do nível adjacente ${ }^{28}$.
No caso $\mathrm{n}^{\circ} .3$ - estenose em múltiplos níveis sem deformidade houve grande concordância quanto à necessidade de descompressão e de realização de artrodese com instrumentação. Foi interessante observar que entre os cirurgiões que optaram pela instrumentação a média de idade foi maior, possivelmente reflexo da experiência profissional frente a esses casos; entretanto, àqueles que não instrumentariam foram a minoria. Os resultados da descompressão cirúrgica em estenose de canal são favoráveis e já bem estabelecidos na literatura por uma série de estudos. A realização da artrodese no tratamento cirúrgico da estenose vem sendo constantemente debatida após a descompressão na ausência de deformidade ou instabilidade, pois a realização da artrodese não apresenta benefícios clínicos, a não ser que seja necessária a remoção das facetas para descompressão ${ }^{29}$. A realização da artrodese na estenose, associada a espondilolistese também é motivo de discussão. Embora uma recente metanálise sobre o tratamento da espondilolistese degenerativa associada à estenose demonstrou que os resultados da realização da artrodese são mais significantes $(p<0,00001)^{30}$. O uso de parafusos pediculares na realização da artrodese aumenta os índices de fusão e os resultados clíni$\cos$ quando feita descompresão e artrodese ${ }^{31-32}$.

\section{CONCLUSÃO}

Após análise dos dados, verificou-se grande concordância quanto às preferências dos cirurgiões de coluna. As diferenças restringem-se à preferência pela instrumentação entre os ortopedistas quando confrontados com casos de hérnia de disco lombar; bem como o tratamento não apenas da clínica neurológica, mas também da deformidade no caso de escoliose degenerativa. A idade dos cirurgiões e o tempo de atividade foram pouco determinantes nas indicações cirúrgicas. Estes dados apontam para uma crescente uniformização no treinamento dos cirurgiões de coluna, restando diferenças que remontam talvez a formação básica que permanece diferenciada em nosso país, com centros de treinamento específicos apenas de ortopedistas ou de neurocirurgiões. Evidentemente, é necessária uma amostragem maior de observadores e também em outras situações clínicas para que se obtenham mais informações. No entanto, este objetivo continuará sendo perseguido.

\section{REFERÊNCIAS}

1. Guadagnoli E, Hauptman PJ, Ayanian JZ, Pashos CL, McNeil BJ, Cleary PD. Variation in the use of cardiac procedures after acute myocardial infarction. N Engl J Med. 1995;333(9):573-8.

2. Hannan EL, Siu AL, Kumar D, Kilburn H Jr, Chassin MR. The decline in coronary artery bypass graft surgery mortality in New York State. The role of surgeon volume. JAMA. 1995;273(3):209-13.
3. Keskimäki I, Aro S, Teperi J. Regional variation in surgical procedure rates in Finland. Scand J Soc Med. 1994;22(2):132-8.

4. Pilote L, Califf RM, Sapp S, Miller DP, Mark DB, Weaver WD, et al. Regional variation across the United States in the management of acute myocardial infarction. GUSTO-1 Investigators. Global Utilization of Streptokinase and Tissue Plasminogen Activator for Occluded Coronary Arteries. N Engl J Med. 1995;333(9):565-72.
5. Wennberg JE, Freeman JL, Shelton RM, Bubolz TA. Hospital use and mortality among Medicare beneficiaries in Boston and New Haven. N Engl J Med. 1989;321(17):1168-73.

6. Katz BP, Freund DA, Heck DA, Dittus RS, Paul JE, Wright J, et al. Demographic variation in the rate of knee replacement: a multiyear analysis. Health Serv Res. 1996;31(2):125-40. 
7. Peterson MG, Hollenberg JP, Szatrowski TP, Johanson NA, Mancuso CA, Charlson ME. Geographic variations in the rates of elective total hip and knee arthroplasties among Medicare beneficiaries in the United States. J Bone Joint Surg Am. 1992;74(10):1530-9.

8. Vitale MG, Krant JJ, Gelijns AC, Heitjan DF, Arons RR, Bigliani LU, et al. Geographic variations in the rates of operative procedures involving the shoulder, including total shoulder replacement, humeral head replacement, and rotator cuff repair. J Bone Joint Surg Am. 1999;81(6):763-72.

9. The Center for the Evaluative Clinical Sciences, Dartmouth Medical School. The Dartmouth Atlas of Musculoskeletal Health Care. The Trustees of Dartmouth College, 2000. Sledge CB. The dartmouth atlas of musculoskeletal health care. J Bone Joint Surg. 2001; 83:1129-30.

10. Volinn E, Mayer J, Diehr P, Van Koevering D, Connell FA, Loeser JD. Small area analysis of surgery for lowback pain. Spine. 1992;17(5):575-81.

11. Keller RB, Soule DN, Wennberg JE, Hanley DF. Dealing with geographic variations in the use of hospitals. The experience of the Maine Medical Assessment Foundation Orthopaedic Study Group. J Bone Joint Surg Am. 1990;72(9):1286-93.

12.Aebi M. Correction of degenerative scoliosis of the lumbar spine. A preliminary report. Clin Orthop Relat Res. 1988;(232):80-6.

13.Herkowitz HN, Kurz LT. Degenerative lumbar spondylolisthesis with spinal stenosis. A prospective study comparing decompression with decompression and intertransverse process arthrodesis. J Bone Joint Surg Am. 1991;73(6):802-8.

14.Herkowitz HN. Spine update. Degenerative lumbar spondylolisthesis. Spine. 1995;20(9):1084-90.

15.Lenke LG, Bridwell KH, Bullis D, Betz RR, Baldus C, Schoenecker PL. Results of in situ fusion for isthmic spondylolisthesis. J Spinal Disord. 1992; 5(4):433-42.
16.Fischgrund JS, Mackay M, Herkowitz HN, Brower R, Montgomery DM, Kurz LT. 1997 Volvo Award winner in clinical studies. Degenerative lumbar spondylolisthesis with spinal stenosis: a prospective, randomized study comparing decompressive laminectomy and arthrodesis with and without spinal instrumentation. Spine. 1997;22(24):2807-12.

17.Bjarke Christensen F, Stender Hansen E, Laursen M, Thomsen K, Bünger $\mathrm{CE}$. Long-term functional outcome of pedicle screw instrumentation as a support for posterolateral spinal fusion: randomized clinical study with a 5-year follow-up. Spine. 2002;27(12):1269-77.

18. Thomsen K, Christensen FB, Eiskjaer SP, Hansen ES, Fruensgaard S, Bünger CE. 1997 Volvo Award winner in clinical studies. The effect of pedicle screw instrumentation on functional outcome and fusion rates in posterolateral lumbar spinal fusion: a prospective, randomized clinical study. Spine. 1997 Dec;22(24):2813-22.

19.Simmons EH, Jackson RP. The management of nerve root entrapment syndromes associated with the collapsing scoliosis of idiopathic lumbar and thoracolumbar curves. Spine. 1979;4(6):533-41.

20.Simmons ED Jr, Simmons EH. Spinal stenosis with scoliosis. Spine. 1992;17(6 Suppl):S117-20.

21. Weber H. Lumbar disc herniation. A controlled, prospective study with ten years of observation. Spine. 1983;8(2):131-40.

22.Frymoyer JW, Matteri RE, Hanley EN, Kuhlmann D, Howe J. Failed lumbar disc surgery requiring second operation. A long-term follow-up study. Spine. 1978;3(1):7-11.

23.Cheng CL, Fang D, Lee PC, Leong JC. Anterior spinal fusion for spondylolysis and isthmic spondylolisthesis. Long term results in adults. J Bone Joint Surg Br. 1989;71(2):264-7.

24.Turner JA, Ersek M, Herron L, Haselkorn J, Kent D, Ciol MA, et al. Patient outcomes after lumbar spinal fusions. JAMA. 1992;268(7):907-11.
25.Fritzell $P$, Hägg $O$, Wessberg $P$, Nordwall A; Swedish Lumbar Spine Study Group. 2001 Volvo Award Winner in Clinical Studies: Lumbar fusion versus nonsurgical treatment for chronic low back pain: a multicenter randomized controlled trial from the Swedish Lumbar Spine Study Group. Spine. 2001;26(23):2521-32.

26.Stoll TM, Dubois G, Schwarzenbach O. The dynamic neutralization system for the spine: a multi-center study of a novel non-fusion system. Eur Spine J. 2002;11 Suppl 2:S170-8.

27.Schmoelz W, Huber JF, Nydegger T, Dipl-Ing, Claes L, Wilke HJ. Dynamic stabilization of the lumbar spine and its effects on adjacent segments: an in vitro experiment. J Spinal Disord Tech. 2003;16(4):418-23.

28. White AH, von Rogov P, Zucherman J, Heiden D. Lumbar laminectomy for herniated disc: a prospective controlled comparison with internal fixation fusion. Spine. 1987;12(3):305-7.

29. Grob D, Humke T, Dvorak J. Degenerative lumbar spinal stenosis. Decompression with and without arthrodesis. J Bone Joint Surg Am. 1995;77(7):1036-41.

30.Mardjetko SM, Connolly PJ, Shott S. Degenerative lumbar spondylolisthesis. A meta-analysis of literature 19701993. Spine. 1994;19(20 Suppl):2256S2265S.

31.Markwalder TM. Surgical management of neurogenic claudication in 100 patients with lumbar spinal stenosis due to degenerative spondylolisthesis. Acta Neurochir (Wien). 1993;120(34):136-42.

32.Zdeblick TA. A prospective, randomized study of lumbar fusion. Preliminary results. Spine. 1993;18(8):983-91.

\section{Correspondência}

Luís Felipe Pamplona Novaes

Departamento de Ortopedia e

Traumatologia

Caixa Postal 6111

CEP: $13081-970$

Campinas (SP), Brasil.

Telefone: +55 $193521-7505$

E-mail: lvisfelipepn@hotmail.com 